\title{
Propuesta integral del sistema de gestión para las buenas prácticas de manufactura del sector cosmético colombiano*
}

\author{
Comprehensive proposal of the management system for good \\ manufacturing practices in the Colombian cosmetic sector
}

\section{Proposta integral do sistema de gestão para as Boas Práticas de Manufatura no setor cosmético colombiano}

Sandra Milena Rodrígue: Labrador"

Recibido: 25 de septiembre de 2017

Revisado: 15 de enero de 2018

Aceptado: 22 de marzo de 2018

INVIMA

\section{RESUMEN}

Actualmente los productores de cosméticos en Colombia se enfrentan con un entorno cada vez más competitivo y exigente; por un lado, para comercializar sus productos en territorio nacional y países de la Comunidad Andina de Naciones deben implementar y cumplir con lo establecido en la legislación, Decisión 516 del 2002 y Resolución 3773 del 2004, pero si desean llegar a países de la Comunidad Europea deben cumplir con las Buenas Prácticas de Manufactura (ISO 22716:2007). Los productores de cosméticos pueden certificar con la autoridad sanitaria, INVIMA, de forma voluntaria, las Buenas Prácticas de Manufactura a través de la Resolución 3774 del 2004 o con un ente certificador la ISO 22716:2007. Este es el panorama del sector de cosméticos en Colombia, que, como respuesta al entorno competitivo, implementan los

\footnotetext{
Artículo de resultado de investigación. DOI: http://dx.doi.org/10.15332/s2145-1389.2018.0001.03

** Magíster en Calidad y Gestión Integral, Universidad Santo Tomás - Convenio ICONTEC. Química industrial, profesional universitario INVIMA. Correo electrónico: milenalabrador@hotmail.com
} 
sistemas de gestión y las normas legales de manera separada. Por lo tanto, un primer paso fue el diseño de una propuesta integral entre la legislación y las normas sobre las Buenas Prácticas de Manufactura del sector cosmético en Colombia; a través de la identificación, comparación, combinación, incorporación y fusión de los requisitos. El contenido de la propuesta fue evaluada por expertos y como resultado final se generó la guía de verificación.

Palabras clave: integración, Buenas Prácticas de Manufactura, producto cosmético, certificación.

\section{ABSTRACT}

Currently, cosmetics producers in Colombia are facing an increasingly competitive and demanding environment; on the one hand, to market their products in national territory and countries of the Andean Community of Nations they must implement and comply with the provisions in the legislation, Decision 516 of 2002 and Resolution 3773 of 2004, but if they wish to reach the countries of the European Community they must comply with the Good Manufacturing Practices (ISO 22716:2007). Cosmetic producers can voluntarily certify with the health authority, INVIMA, the Good Manufacturing Practices through Resolution 3774 of 2004 or with a certifying body the ISO 22716:2007. This is the panorama of the cosmetics sector in Colombia, which, in response to the competitive environment, implements management systems and legal regulations separately. Therefore, a first step was the design of an comprehensive proposal between the legislation and the standards on Good Manufacturing Practices of the cosmetic sector in Colombia; through the identification, comparison, combination, incorporation and merging of the requirements. The content of the proposal was evaluated by experts and as a final result the verification guide was produced.

Keywords: Integration, Good Manufacturing Practices, cosmetic product, certification.

\section{RESUMO}

Atualmente, os produtores de cosméticos na Colômbia se enfrentam com um ambiente cada vez mais competitivo e exigente; Por um lado, para comercializar seus produtos em território nacional e os países da Comunidade Andina de Nações devem implementar e cumprir as disposições na legislação, a Decisão 516 de 2002 e a Resolução 3773 de 2004, mas se desejam chegar a países da Comunidade Européia devem cumprir com as Boas Práticas de manufatura (ISO 22716: 2007). Os produtores de cosméticos podem certificar com a autoridade sanitária, INVIMA,de forma voluntária, as Boas Práticas de manufatura através da Resolução 3774 de 2004 ou com um órgão certificador da ISO 22716: 2007. Este é o panorama do setor de cosméticos na Colômbia, que, em resposta ao ambiente competitivo, implementam sistemas de gestão e as normais legais de forma separada. Portanto, um primeiro passo é o desenho de uma proposta integral entre a legislação e as normas sobre as Boas Práticas de manufatura do setor cosmético na Colômbia; através da identificação, comparação, combinação, incorporação e fusão dos requisitos. O conteúdo da proposta foi avaliado por especialistas e, como resultado final, Foi gerado o guia de verificação.

Palavras-chave: integração, Boas Práticas de manufatura, produto cosmético, certificação. 


\section{INTRODUCCIÓN}

Las Buenas Prácticas de Manufactura Cosméticos a través del tiempo ha tenido su evolución, tal como se observa en la figura 1, iniciándose el recorrido en 1995, cuando el Consejo de Europa a través del comité de expertos en productos cosméticos, publican las directrices de las Buenas Prácticas de Producción para Productos Cosméticos (BPPC), basada en "recomendaciones prácticas que tiene como objetivo eliminar y prevenir las deficiencias de calidad en la producción de productos cosméticos, alentando a las empresas a establecer su sistema de calidad, pero respetando la seguridad del personal y del medio ambiente" (Conseil de l'Europe, 1995, p. 7).

Los países miembros de la Comunidad Andina de Naciones (CAN), en aras de armonizar las legislaciones internas referente a productos cosméticos, crean la Decisión 516, la cual entra en vigencia el 15 de marzo de 2002, en su anexo 2 adoptan la Norma Técnica Armonizada de Buenas Prácticas de Manufactura
Cosmética, siendo esta de obligatorio cumplimiento para optar por el certificado de capacidad de producción (Comisión de la Comunidad Andina, 2002).

Por lo tanto, el 10 de noviembre de 2004 el Ministerio de Protección Social como ente normalizador, emite la Resolución 3773 en donde se adopta la guía de capacidad para la fabricación de productos cosméticos, dando cumplimiento a lo establecido en el artículo 29 de la Decisión 516; para esta misma fecha se emite la Norma Técnica Armonizada de Buenas Prácticas de Manufactura Cosmética (Ministerio de Protección Social, 2004a) y la guía de verificación (Ministerio de Protección Social, 2004b), a través de la Resolución 3774, su certificación y cumplimiento es de carácter voluntario por parte de los fabricantes de productos cosméticos en Colombia.

International Organization for Standardization (ISO), publica la norma ISO 22716:2007, la cual proporciona las directrices para las buenas prácticas de manufactura del sector cosmético, en cuanto a los aspectos de

Figura 1. Evolución de las Buenas Prácticas de Manufactura Cosméticos

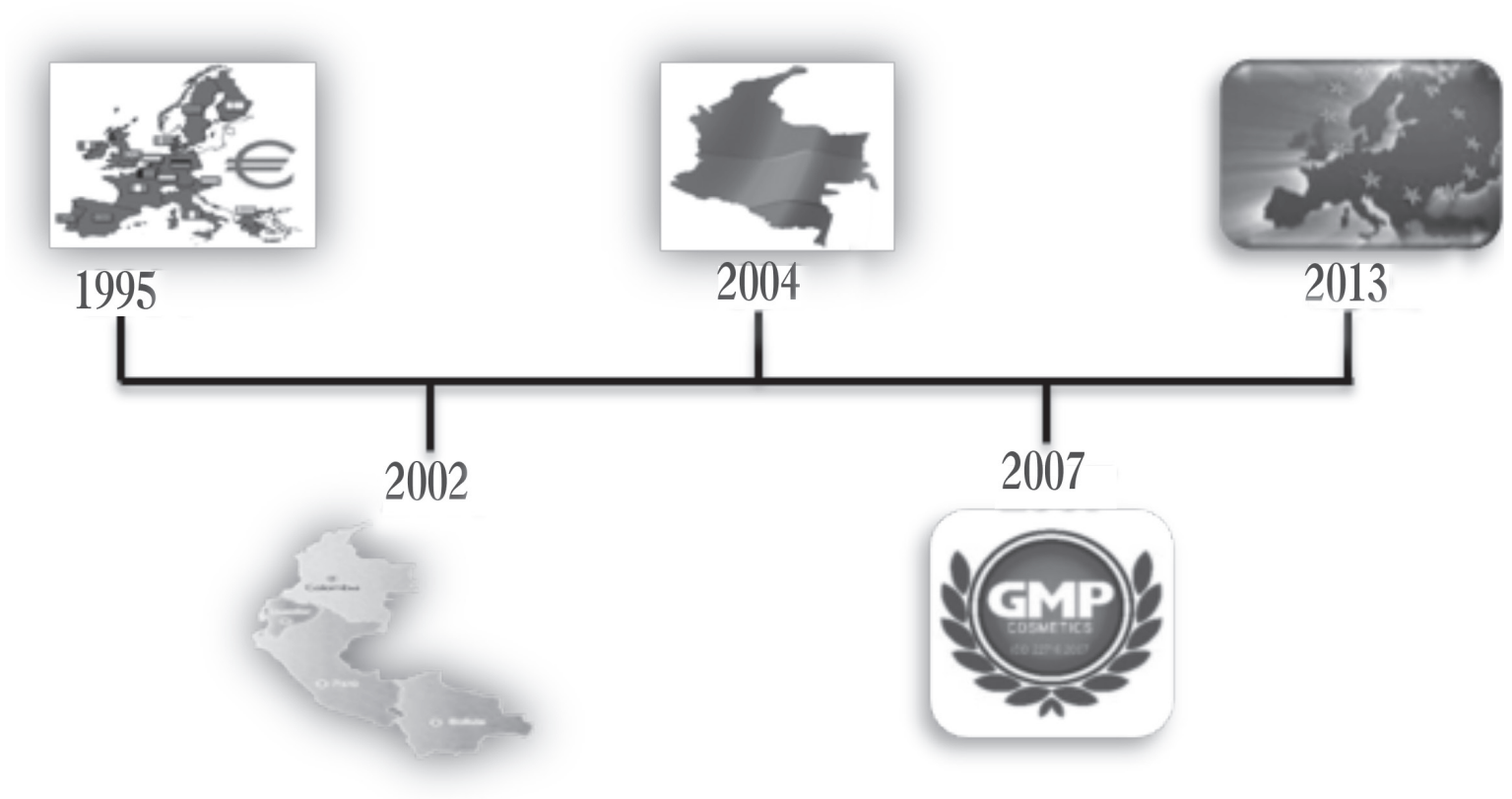

Fuente: elaboración propia. 
producción, control, almacenamiento y envío, abarcando la gestión de los factores humanos, técnicos y administrativos que afectan la calidad del producto; no cubre aspectos de seguridad para el personal contratado en la planta, ni cubre aspectos de la protección del medio ambiente, ya que estas son responsabilidades inherentes de la empresa, tan poco cubre las etapas de investigación, de desarrollo y distribución de productos terminados. Esta norma fue revisada y confirmada en 2017, por tanto, esta versión sigue siendo actual.

La Unión Europea mediante los organismos de normalización, armonizaron la norma ISO 22716 y como resultado se obtuvo la Norma UNE-EN ISO 22716, publicada en el Diario Oficial de la Unión Europea (D.O.U.E), convirtiéndose en una norma de OBLIGATORIO cumplimiento desde el 11 de julio de 2013 (Agencia Valencia de Salut, 2013).

Los productos cosméticos fabricados en Colombia son vendidos anualmente en países europeos, convirtiéndose en un mercado objetivo de exportación. En el año 2015, Colombia exportó a la Unión Europea un valor de USD 2.5 millones en productos cosméticos (Programa Safe+, 2016). Así mismo, uno de los objetivos sectoriales para el 2032, es el de multiplicar las exportaciones al menos 7 veces hasta llegar a exportar el $27 \%$ de la producción (Cámara de la Industria Cosmética y de Aseo de la ANDI, 2013); objetivo muy alineado con la visión establecida por el estado colombiano dentro de su política nacional de competitividad y productividad, Conpes 3527, en la cual Colombia será uno de los tres países más competitivos de América Latina (Consejo Nacional de Política Económica y Social, 2008).

También es relevante mencionar que en este momento no existe una única definición para el término de integración; por lo tanto, dentro de la UNE 66177 se encuentra que INTEGRAR es "la acción y efecto de aunar dos o más políticas, conceptos, corrientes, etc., divergentes entre sí, fusionándolos en una sola que lo sintetice" (Asociación Española de Normalización y Certificación, 2005, p. 5); hallándose a su vez que la INTEGRACIÓN es "el proceso de vincular diferentes sistemas de gestión estandarizados dentro de un único sistema de gestión con recursos comunes, con el objetivo de mejorar la satisfacción de las partes interesadas" (Bernardo, Casadesus, Karapetrovic y Heras, 2009, p. 744) y de igual forma "para cada organización el concepto de integración varía de acuerdo al contexto y a la definición particular en la que se desarrollan cada uno de sus procesos" (Bonilla y Martínez, 2015, p. 2). Por lo tanto, al definir la palabra INTEGRACIÓN, no es posible dejar de mencionar verbos tales como armonizar, fusionar y unir; al mismo tiempo frases como: constituir un todo, completar un todo con las partes que faltaban (Llanes-Font, Isaac, Moreno y García, 2014).

En el 2017, Colombia contaba con 473 empresas productoras de cosméticos, certificadas por el INVIMA en cuanto a su capacidad de producción, conforme a lo establecido en la legislación sanitaria vigente, Decisión 516 de 2002 y Resolución 3773 de 2004 (INVIMA, 2018a); pero tan solo el $3 \%$ de dichas empresas se encuentran certificadas en las Buenas Prácticas de Manufactura Cosmética de acuerdo con la Resolución 3774 de 2004 (INVIMA, 2018b). Desde este panorama, los fabricantes de productos cosméticos en Colombia duplican sus esfuerzos y la inversión que realizan, al implementar y cumplir de manera separada e independiente, la legislación nacional e implementar las Buenas Prácticas de Manufactura; por lo tanto, se detecta la necesidad de diseñar una propuesta integral entre la Decisión 516 del 2002, Resolución 3773 del 2004, la Resolución 3774 del 2004 y ISO 22716:2007, convirtiéndose en el objetivo principal de la investigación. 


\section{METODOLOGÍA}

En la figura 2 se ilustra la metodología desarrollada durante la investigación, la cual tiene un enfoque mixto, ya que su estructura se desenvuelve de manera sistémica, entre la recolección y el análisis de los datos cualitativos y cuantitativos obtenidos en la integración de los elementos que componen las normas actuales de las Buenas Prácticas de Manufactura del sector cosmético en Colombia.

El diseño es exploratorio secuencial en la modalidad derivativa, iniciando con la recolección y análisis de los datos cualitativos obtenidos, a través de la creación de matrices comparativas entre la legislación colombiana actual y vigente de las Buenas Prácticas de Manufactura del sector cosmético, el anexo 2 de la Decisión 516 de 2002, la Resolución 3773 de 2004, la Resolución 3774 de 2004 en conjunto con la norma internacional ISO 22716:2007, que origina la construcción y el diseño de la propuesta integral para el sistema de gestión de las Buenas Prácticas de Manufactura del sector cosmético colombiano, dicho diseño se desarrolló en las etapas, que se observan en la figura 3 :
Figura 2. Metodología desarrollada

Enfoque

MIXTO

Diseño

EXPLORATORIO

SECUENCIAL

Codificación selectiva

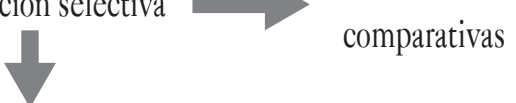

Evaluación por los expertos

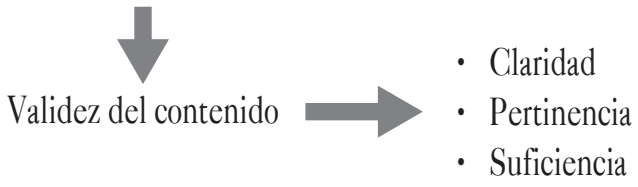

Fuente: elaboración propia.

El contenido de la propuesta fue evaluado cuantitativamente en los criterios de claridad, pertinencia y suficiencia en escalas Likert.

Fiǵura 3. Etapas desarrolladas en el diseño de la propuesta integral

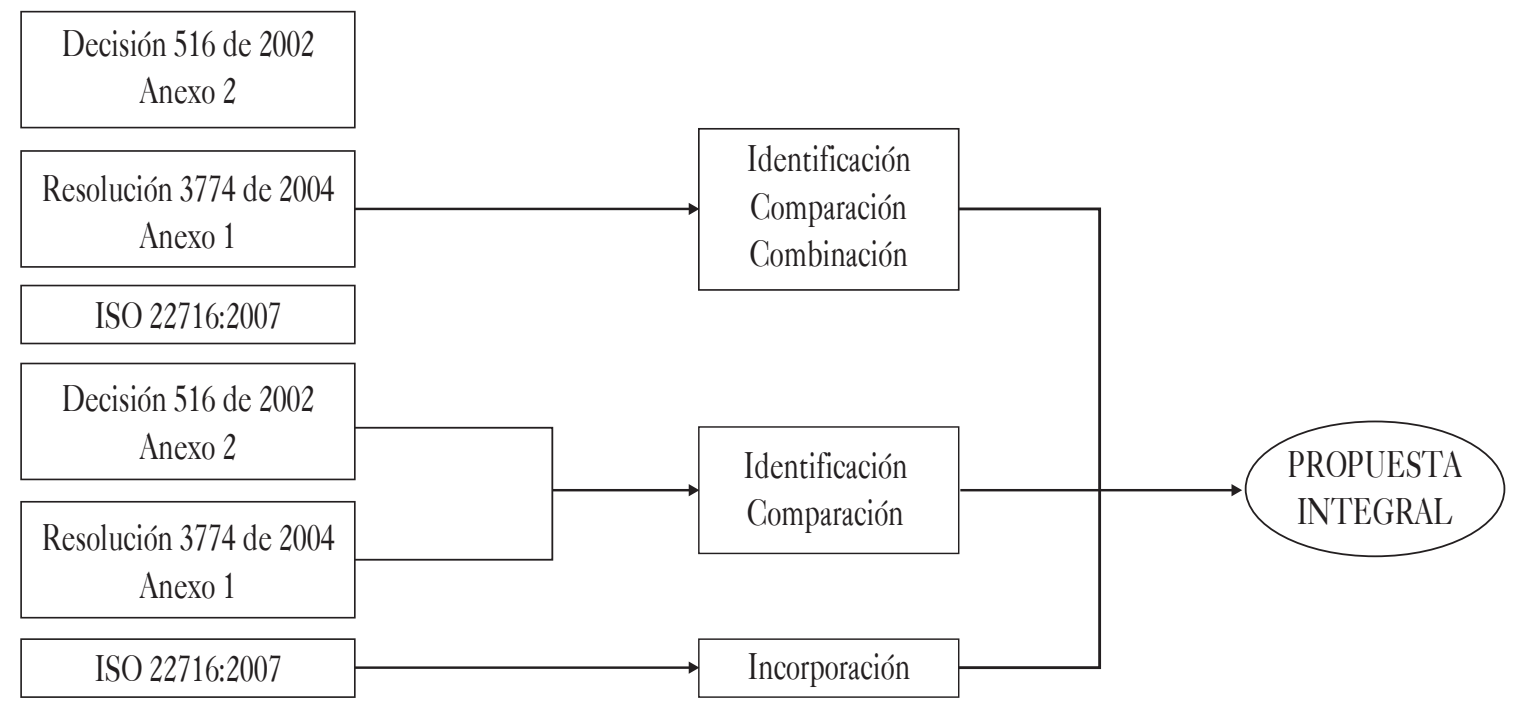

Fuente: elaboración propia. 
La evaluación fue realizada en una muestra no probabilística, de ocho expertos, los cuales son profesionales en Química Farmacéutica e Ingeniería Química, con experiencia laboral en la certificación de las normas anexo 2 de la Decisión 516 de 2002, la Resolución 3773 de 2004, la Resolución 3774 de 2004 y en su efecto cuentan con la experiencia en el sector cosmético y manufacturero, así como con los estudios en la norma ISO 22716.

Después de aplicar las evaluaciones se tabularon, analizaron e interpretaron los resultados obtenidos, a través de la estadística descriptiva (distribución de frecuencias) y posteriormente se realizó el respectivo ajuste a la propuesta integral para el sistema de gestión con enfoque integral de las Buenas Prácticas de Manufactura del sector cosmético en Colombia.

Finalmente, "la mezcla mixta ocurre cuando se conecta el análisis cualitativo de los datos y la recolección de datos cuantitativos. La interpretación final es producto de la integración y comparación de resultados cualitativos y cuantitativos" (Hernández, Fernández y Baptista, 2010, p. 564). Se procedió a realizar la guía de verificación de la propuesta integral para el sistema de gestión entre el anexo 2 de la Decisión 516 del 2002, la Resolución 3773 del 2004, la Resolución 3774 del 2004 y la norma ISO 22716:2007 para el cumplimiento de las Buenas Prácticas de Manufactura del sector cosmético en Colombia.

\section{RESULTADOS Y DISCUSIÓN}

En el primer resultado cualitativo de las matrices comparativas, se evidenció la estructura lógica, semejante y equivalente entre el anexo 2 de la Decisión 516 del 2002, la Resolución 3773 del 2004, la Resolución 3774 del 2004 y la norma ISO 22716:2007 para las Buenas Prácticas de Manufactura del sector cosmético en Colombia; obteniéndose los quince capítulos que conforma la propuesta integral del sistema de gestión para las Buenas Prácticas de Manufactura del sector cosmético colombiano.

El capítulo 1. Personal, quedó constituido por los aspectos de: orgaanización, responsabilidades clave, formación e higiene y salud del personal. De acuerdo con la integración se obtuvo el siguiente resultado:

Figura 4. Resultados obtenidos en el capítulo 1. Personal

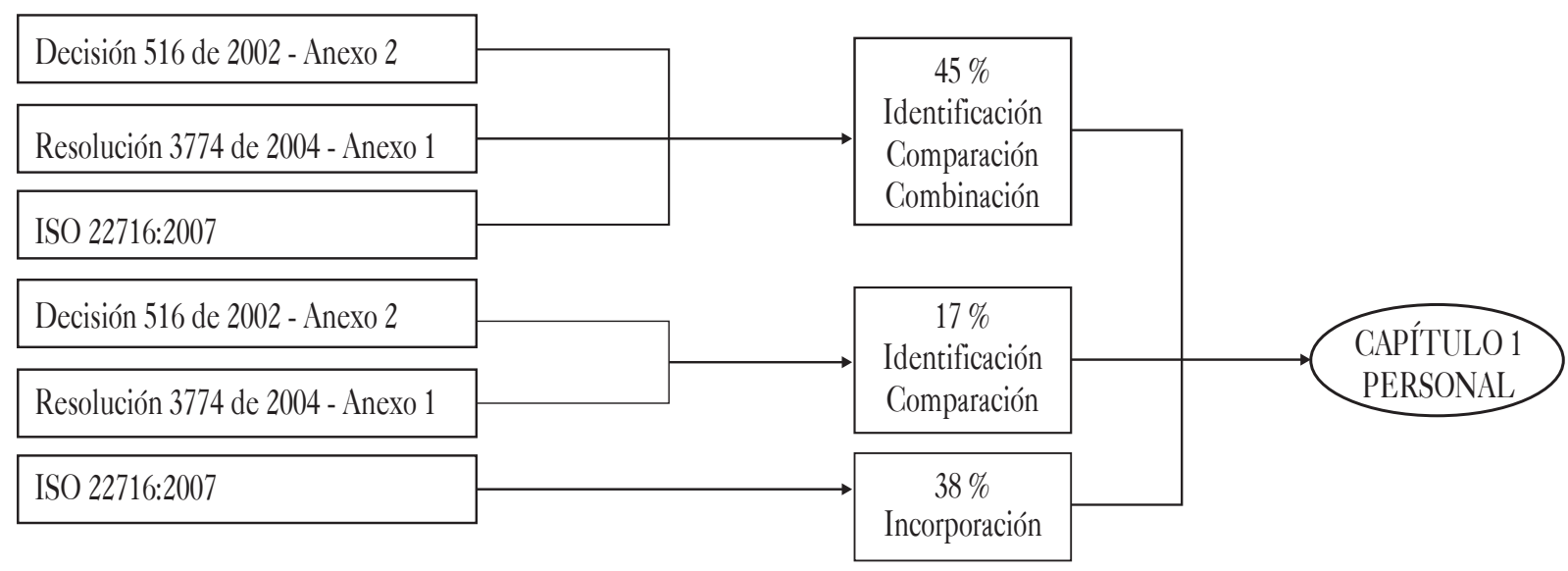

Fuente: elaboración propia. 
La propuesta inicial para el capítulo 1 estaba conformada por 29 requisitos, una vez fue evaluada por los expertos quedó un total de 28 requisitos, por lo que un experto consideró pertinente incorporar el requisito 1.5.1.5 con el requisito 1.5.1.4 por tratarse del mismo tema de las prohibiciones de no comer, beber, masticar y fumar en áreas productivas.

En cuanto a los resultados obtenidos de la evaluación por los expertos para este capítulo, se concentraron en la escala 4 para los criterios de claridad, pertinencia y suficiencia, tal como se muestra en la tabla 1 :

Tabla 1. Resultados obtenidos para el capítulo 1. Personal

\begin{tabular}{|c|c|c|c|}
\hline \multicolumn{4}{|c|}{ CAPÍTULO 1. PERSONAL } \\
\hline Escala & Claridad & Pertinencia & Suficiencia \\
\hline 1 & $0.4 \%$ & $2.2 \%$ & $2.2 \%$ \\
\hline 2 & $3.9 \%$ & $1.3 \%$ & $2.2 \%$ \\
\hline 3 & $14.7 \%$ & $3.9 \%$ & $6.9 \%$ \\
\hline 4 & $81.0 \%$ & $92.7 \%$ & $88.8 \%$ \\
\hline
\end{tabular}

Fuente: elaboración propia.
El capítulo 2. Instalaciones, quedó constituido por los aspectos de: tipos de área, espacios, flujo, pisos, paredes, cielos rasos y ventanas, vestieres, instalaciones sanitarias y de lavado, iluminación, ventilación, tuberías, drenajes y conductos, limpieza y sanitización, mantenimiento y control de plagas; conformado por un total de 35 requisitos y obteniéndose como resultado de la integración, el siguiente:

Es de recalcar que los requisitos 2.6.2 y 2.6.4, tratan aspectos que no son contemplados por ninguna de las normas actuales y que la autora por su experiencia incluyó en la propuesta, como lo son la ubicación de los servicios sanitarios y las áreas de lavado para los materiales, equipos, utensilios y accesorios provenientes de las áreas productivas; por ser áreas que pueden representar un riesgo de contaminación cruzada en el desarrollo de las Buenas Prácticas de Manufactura. Estos requisitos fueron evaluados por los expertos.

Figura 5. Resultados obtenidos en el capítulo 2. Instalaciones

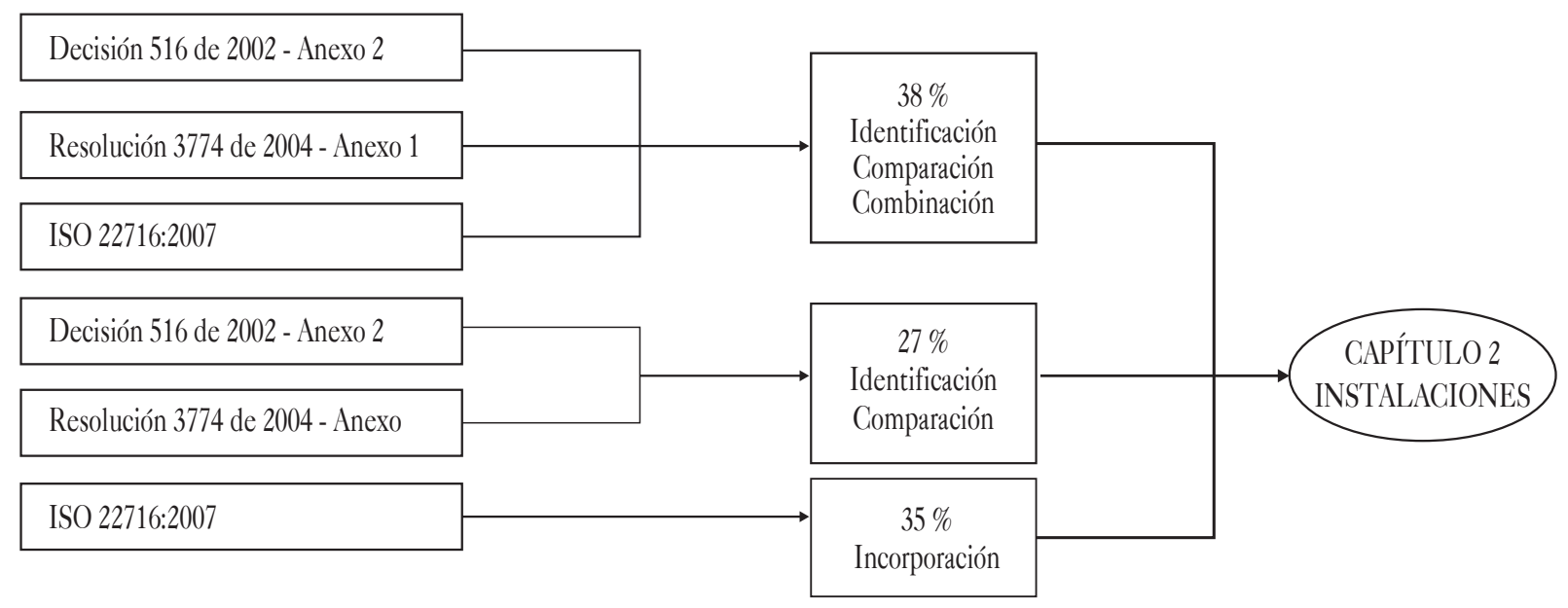

Fuente: elaboración propia. 
Tabla 2. Resultados obtenidos para los requisitos 2.6 .2 y 2.6.4

\begin{tabular}{|c|c|c|c|c|c|c|}
\hline \multicolumn{4}{|c|}{ REQUISITO 2.6.2 } & \multicolumn{3}{c|}{ REQUISITO 2.6.4 } \\
\hline Esca la & Claridad & Pertinencia & Suficiencia & Claridad & Pertinencia & Suficiencia \\
\hline 1 & $0 \%$ & $0 \%$ & $0 \%$ & $0 \%$ & $0 \%$ & $0 \%$ \\
\hline 2 & $0 \%$ & $0 \%$ & $12.5 \%$ & $0 \%$ & $0 \%$ & $12.5 \%$ \\
\hline 3 & $12.5 \%$ & $0 \%$ & $0 \%$ & $12.5 \%$ & $0 \%$ & $0 \%$ \\
\hline 4 & $87.5 \%$ & $100 \%$ & $87.5 \%$ & $87.5 \%$ & $100 \%$ & $87.5 \%$ \\
\hline
\end{tabular}

Fuente: elaboración propia.

Los resultados obtenidos de la evaluación por los expertos para este capítulo, se concentraron en la escala 4 para los criterios de claridad, pertinencia y suficiencia.

Tabla 3. Resultados obtenidos para el capítulo 2. Instalaciones
El capítulo 3. Equipos, está constituido por los aspectos de: diseño del equipo, instalación, calibración, limpieza y sanitización, mantenimiento, autorizaciones y sistemas de soporte, de acuerdo con la integración se obtuvo el siguiente resultado:

\begin{tabular}{|c|c|c|c|}
\hline \multicolumn{4}{|c|}{ CAPÍTULO 2. INSTALACIONES } \\
\hline Escala & Claridad & Pertinencia & Suficiencia \\
\hline 1 & $0.7 \%$ & $1.4 \%$ & $0.7 \%$ \\
\hline 2 & $3.2 \%$ & $0.4 \%$ & $2.9 \%$ \\
\hline 3 & $14.6 \%$ & $3.6 \%$ & $3.9 \%$ \\
\hline 4 & $81.4 \%$ & $94.6 \%$ & $92.5 \%$ \\
\hline
\end{tabular}

Fuente: elaboración propia.

Figura 6. Resultados obtenidos en el capítulo 3. Equipos

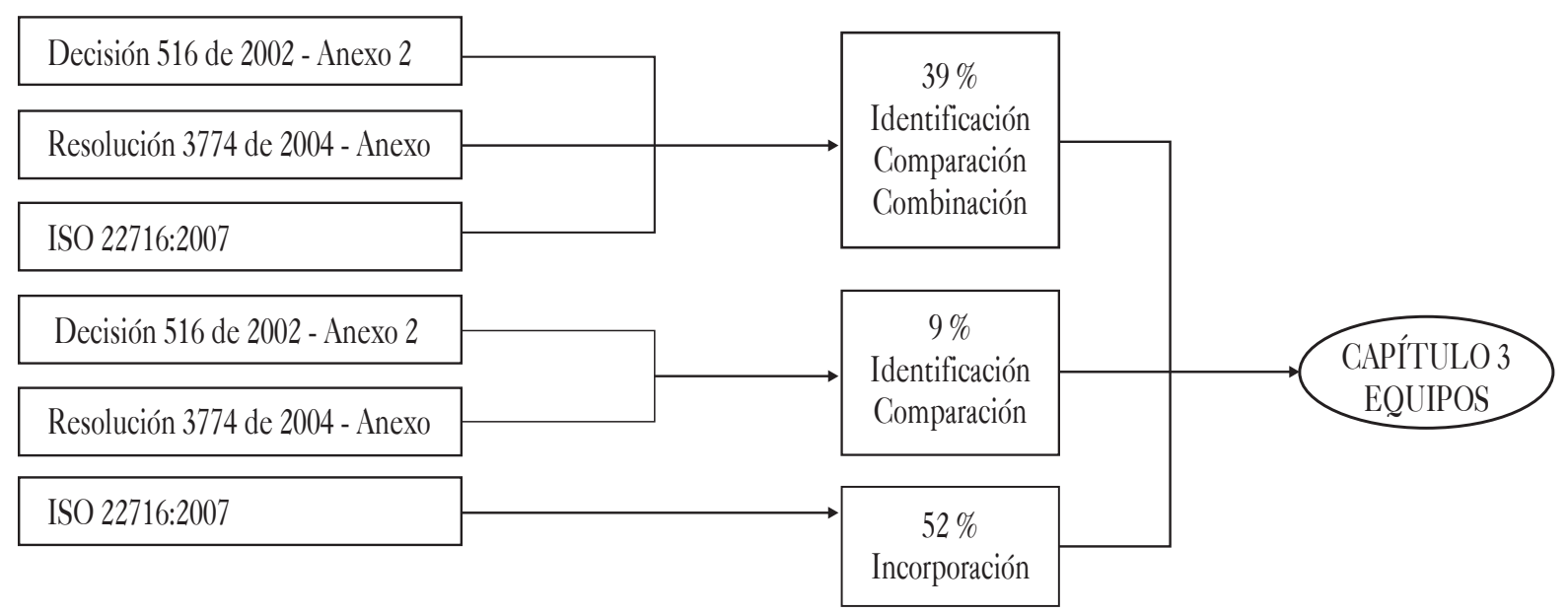

Fuente: elaboración propia. 
La propuesta inicial para el capítulo 3 estaba conformada por 23 requisitos, una vez fue evaluada por los expertos quedó un total de 21 requisitos, debido a que un experto realizó la observación de que el requisito 3.4.3 se podía incorporar con el requisito 3.4.2 por tratarse de los resultados de la calibración que se encuentran por fuera de los criterios de aceptación, así mismo el requisito 3.2.3 correspondiente a los recipientes del producto a granel se unificó con el numeral 5.2.6.1 por tratarse de condiciones específicas de almacenamiento del producto a granel, esto se da como resultado de la observación que realizó un evaluador al manifestar que este requisito no correspondía al capítulo de equipos.

Los resultados obtenidos de la evaluación por los expertos para este capítulo, se concentraron en la escala 4 para los criterios de claridad, pertinencia y suficiencia.

Tabla 4. Resultados obtenidos para el capítulo 3. Equipos

\begin{tabular}{|c|c|c|c|}
\hline \multicolumn{4}{|c|}{ CAPÍTULO 3. EQUIPOS } \\
\hline Escala & Claridad & Pertinencia & Suficiencia \\
\hline 1 & $0.5 \%$ & $1.6 \%$ & $1.6 \%$ \\
\hline 2 & $1.6 \%$ & $2.7 \%$ & $2.2 \%$ \\
\hline 3 & $4.3 \%$ & $0 \%$ & $0.5 \%$ \\
\hline 4 & $93.5 \%$ & $95.7 \%$ & $95.7 \%$ \\
\hline
\end{tabular}

Fuente: elaboración propia.

Figura 7. Resultados obtenidos en el capítulo 4. Materias primas y materiales de envase y empaque

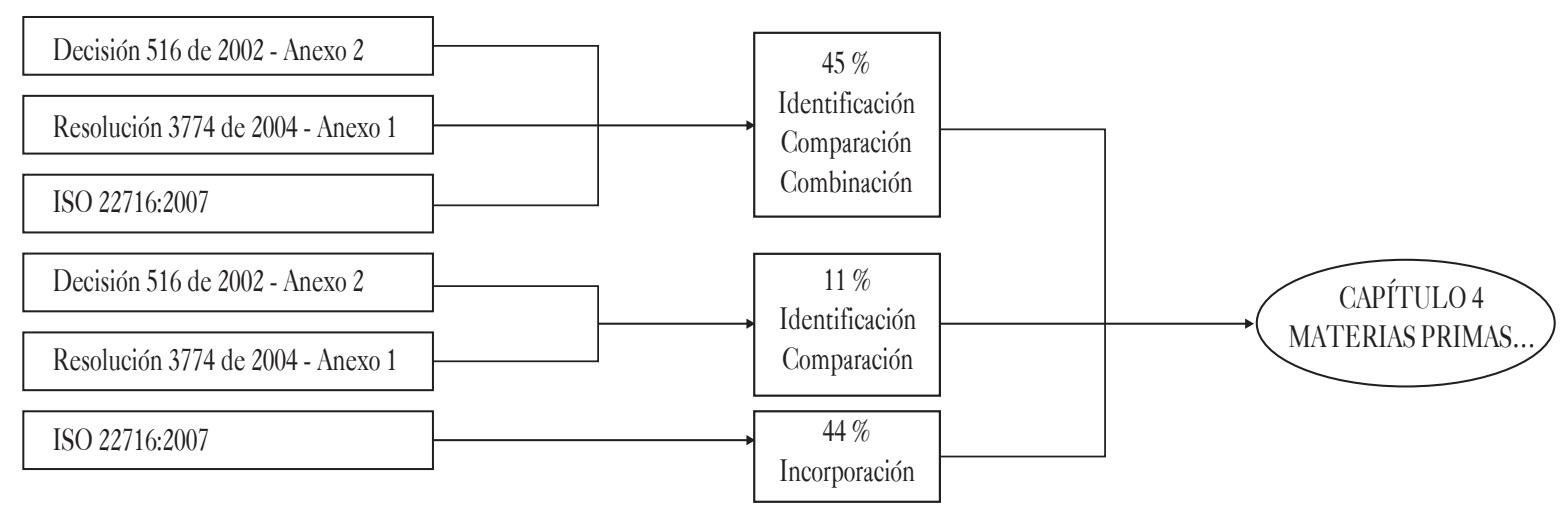

Fuente: elaboración propia.
El capítulo 4. Materias primas y materiales de envase y empaque, quedó constituido por los aspectos de: compras, recepción, identificación y estado, liberación, almacenamiento, reevaluación y calidad del agua utilizada en la producción. La propuesta inicial para el capítulo 4 estaba conformada por 27 requisitos, un evaluador realizó la observación de que el numeral 4.2.1 no era un requisito sino un concepto, por cuanto se trataba del fundamento de la compra de materias primas, materiales de envase y empaque, se procedió a incorporarlo dentro del numeral 4.1. Principios, dando así un total de 26 requisitos. De acuerdo con la integración se obtuvo el siguiente resultado: 
Los resultados obtenidos de la evaluación por los expertos para este capítulo, se concentraron en la escala 4 para los criterios de claridad, pertinencia y suficiencia.

Tabla 5. Resultados obtenidos para el capítulo 4. Materias primas y materiales de envase y empaque

\begin{tabular}{|c|c|c|c|}
\hline \multicolumn{4}{|c|}{ CAPÍTULO 4. MATERIAS PRIMAS Y MATERIALES } \\
DE ENVSE Y EMPAQUE \\
\hline Escala & Claridad & Pertinencia & Suficiencia \\
\hline 1 & $0 \%$ & $0.9 \%$ & $0.5 \%$ \\
\hline 2 & $1.9 \%$ & $0.5 \%$ & $1.9 \%$ \\
\hline 3 & $3.2 \%$ & $0.9 \%$ & $2.3 \%$ \\
\hline 4 & $94.9 \%$ & $97.7 \%$ & $95.4 \%$ \\
\hline
\end{tabular}

Fuente: elaboración propia.

El capítulo 5. Producción, quedó constituido por los subcapítulos de Operaciones de fabricación y Operaciones de envase y acondicionamiento, los cuales están conformados por los aspectos de: disponibilidad de los documentos pertinentes, verificaciones iniciales, asignación del número de lote, identificación de las operaciones en proceso, control en proceso, almacenamiento del producto a granel, devolución al almacén de materias primas, verificaciones del equipo de control en línea, devolución al almacén de materiales de envase y empaque, identificación y manejo de trabajos continuos.

Por lo tanto, el capítulo 5. Producción quedó conformado por un total de 35 requisitos, aunque la propuesta inicial que fue sometida a la evaluación por los expertos era de 38 requisitos, teniendo en cuenta la observación constante dada por un evaluador de que se estaba repitiendo lo mismo, se realizó la unificación del requisito 5.3.3.2 con el 5.2.3.1 por tratarse de la asignación de un número de lote a cada uno de los lotes fabricados del granel y del producto terminado. Por otro lado, se unificó en un solo numeral 5.3.1 los requisitos 5.3.1.1 y 5.3.1.2, por abordar los aspectos sobre la disponibilidad de los documentos pertinentes. De acuerdo con la integración se obtuvo el siguiente resultado:

Figura 8. Resultados obtenidos en el capítulo 5. Producción

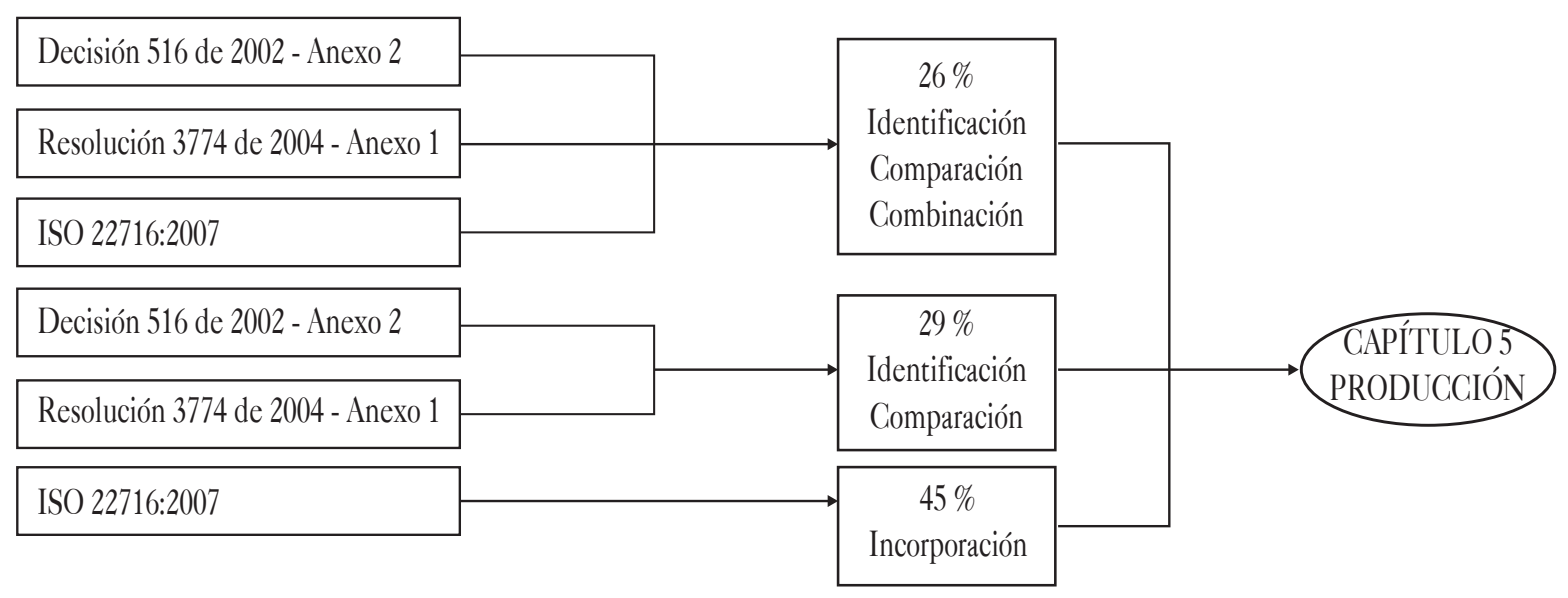

Fuente: elaboración propia. 
Los resultados obtenidos de la evaluación por los expertos para este capítulo, se concentraron en la escala 4 para los criterios de claridad, pertinencia y suficiencia.

Tabla 6. Resultados obtenidos para el capítulo 5. Producción
El capítulo 6. Productos terminados, quedó constituido por los aspectos de: liberación, almacenamiento, despachos, y devoluciones, de acuerdo con la integración se obtuvo el siguiente resultado:

\begin{tabular}{|c|c|c|c|}
\hline \multicolumn{4}{|c|}{ CAPÍTUL0 5. PRODUCGIÓN } \\
\hline Escala & Claridad & Pertinencia & Suficiencia \\
\hline 1 & $0.7 \%$ & $0.7 \%$ & $0.7 \%$ \\
\hline 2 & $1.3 \%$ & $0.7 \%$ & $0.3 \%$ \\
\hline 3 & $10.5 \%$ & $0.3 \%$ & $10.2 \%$ \\
\hline 4 & $87.5 \%$ & $98.4 \%$ & $88.8 \%$ \\
\hline
\end{tabular}

Fuente: elaboración propia.

Figura 9. Resultados obtenidos en el capítulo 6. Productos terminados

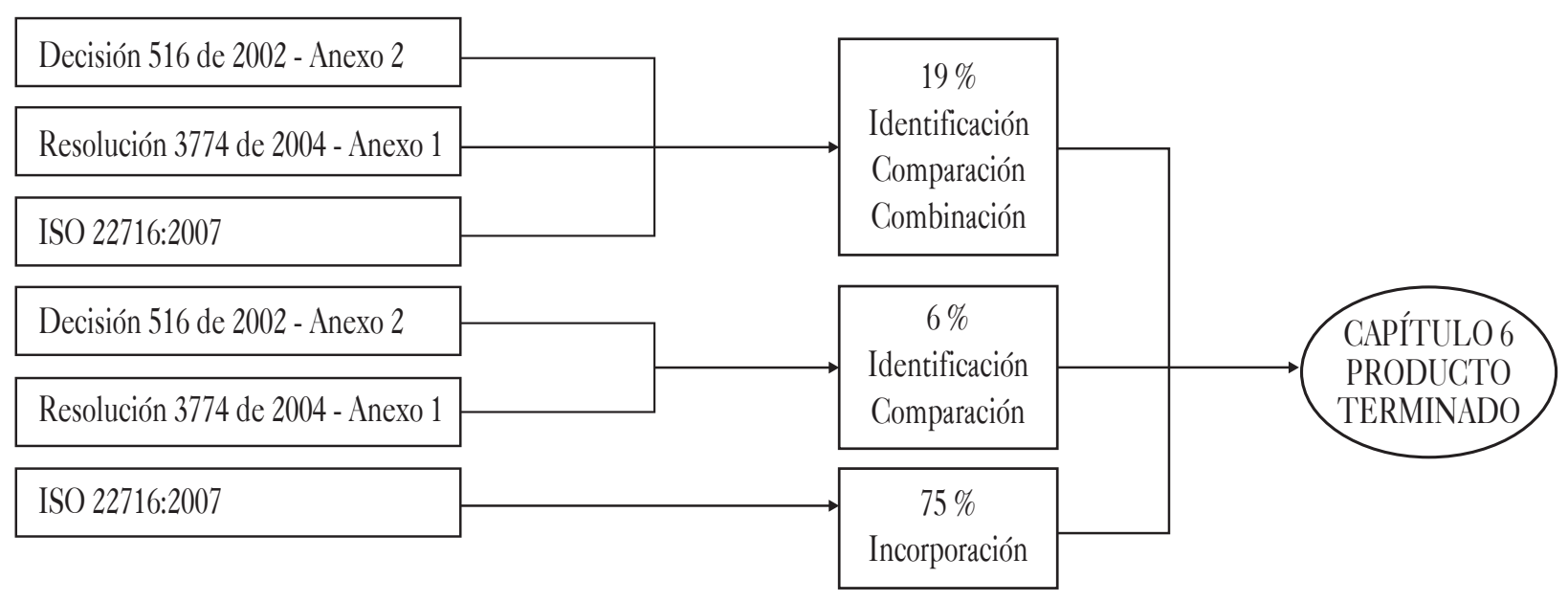

Fuente: elaboración propia.

La propuesta inicial del capítulo 6 estaba constituida por 16 requisitos, una vez fue evaluada por los expertos quedó un total de 15 requisitos, por cuanto se unificó el requisito 6.4.3 con el requisito 6.4.2 por relacionarse con las precauciones y las medidas para mantener la calidad del producto terminado durante el despacho.

Los resultados obtenidos de la evaluación por los expertos para este capítulo se concentraron en la escala 4 para los criterios de claridad, pertinencia y suficiencia.
Tabla 7. Resultados obtenidos para el capítulo 6. Productos terminados

\begin{tabular}{|c|c|c|c|}
\hline \multicolumn{4}{|c|}{ CAPÍTUL0 6. PRODUCTOS TERMINADOS } \\
\hline Escala & Claridad & Pertinencia & Suficiencia \\
\hline 1 & $0.8 \%$ & $1.6 \%$ & $0.8 \%$ \\
\hline 2 & $0 \%$ & $3.1 \%$ & $3.1 \%$ \\
\hline 3 & $9.4 \%$ & $2.3 \%$ & $3.9 \%$ \\
\hline 4 & $89.8 \%$ & $93.0 \%$ & $92.2 \%$ \\
\hline
\end{tabular}

Fuente: elaboración propia. 
El capítulo 7. Laboratorio de control de calidad, quedó constituido por los aspectos de: métodos de ensayo, criterios de aceptación, resultados, resultados fuera de especificación, reactivos, soluciones, patrones de referencia y medios de cultivos, muestreo y retención de la muestra; inicialmente este capítulo estaba conformado por un total de 21 requisitos, aunque la propuesta inicial que fue sometida a la evaluación por los expertos era de 22 requisitos, por cuanto se unificó en un solo numeral 7.4 los requisitos 7.4.1 y 7.4.2, por abordar los aspectos de resultados obtenidos. De acuerdo con la integración se obtuvo el siguiente resultado:

Figura 10. Resultados obtenidos en el capítulo 7.

Laboratorio de control de calidad

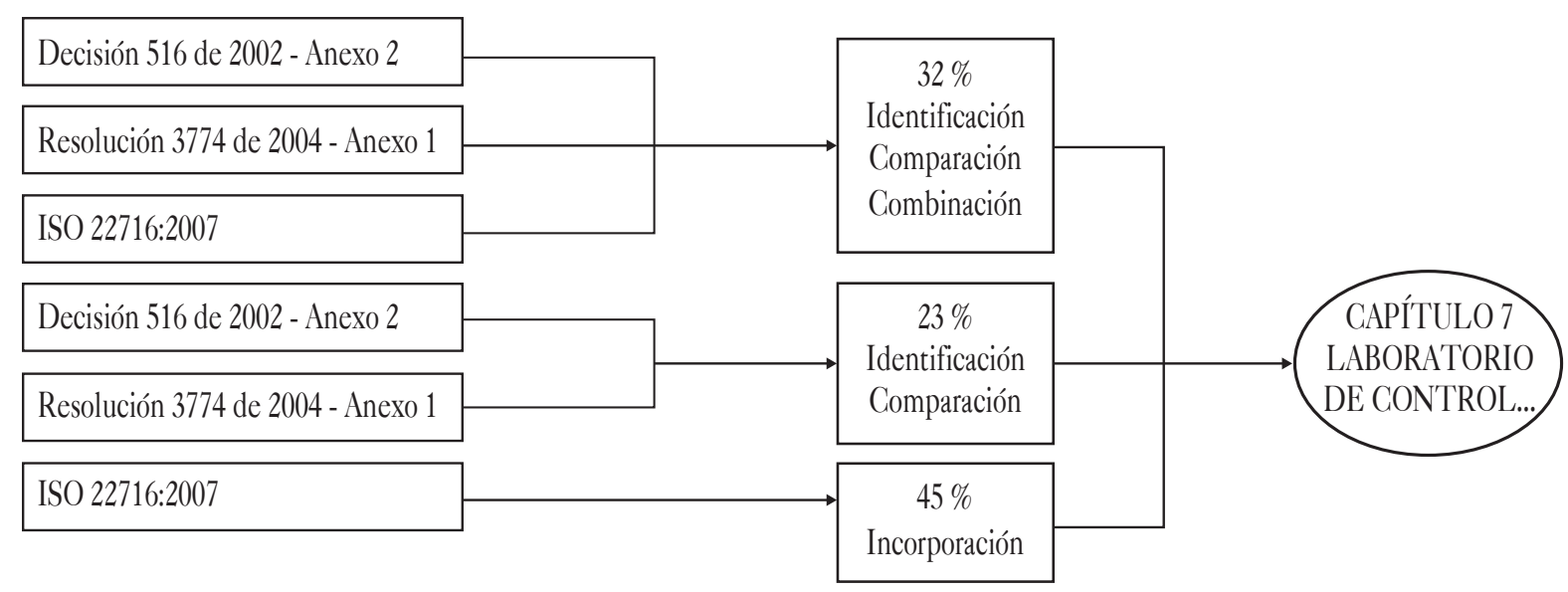

Fuente: elaboración propia.

Los resultados obtenidos de la evaluación por los expertos para este capítulo, se concentraron en la escala 4 para los criterios de claridad, pertinencia y suficiencia.

Tabla 8. Resultados obtenidos para el capítulo 7. Laboratorio de control de calidad

\begin{tabular}{|c|c|c|c|}
\hline \multicolumn{4}{|c|}{ CAPÍTULO 7. LABORATORIO DE CONTROL } \\
DE CALIDAD \\
\hline Escala & Claridad & Pertinencia & Suficiencia \\
\hline 1 & $0 \%$ & $0.6 \%$ & $0.6 \%$ \\
\hline 2 & $1.7 \%$ & $0 \%$ & $1.1 \%$ \\
\hline 3 & $9.7 \%$ & $0 \%$ & $2.8 \%$ \\
\hline 4 & $88.6 \%$ & $99.6 \%$ & $95.5 \%$ \\
\hline
\end{tabular}

Fuente: elaboración propia.
El capítulo 8. Tratamiento del producto que está fuera de especificación, quedó constituido por los aspectos de: productos terminados, productos a granel, materias primas y materiales de envase y empaque rechazados y productos terminados y productos a granel reprocesados; conformado por un total de 5 requisitos, los cuales corresponden a los requisitos incorporados de la ISO 22716:2007, por cuanto la Decisión 516 de 2002 y la Resolución 3774 de 2004 no contemplan el tratamiento del producto que está fuera de especificación.

Los resultados obtenidos por la evaluación de los expertos para este capítulo, se concentraron en la escala 4 para los criterios de claridad, pertinencia y suficiencia. 
Tabla 9. Resultados obtenidos para el capítulo 8. Tratamiento del producto que está fuera de especificación

\begin{tabular}{|c|c|c|c|}
\hline \multicolumn{4}{|c|}{$\begin{array}{c}\text { CAPÍTULO 8. TRATAMIENTO DEL PRODUCTO } \\
\text { QUE ESTÁ FUERA DE ESPECIFICACIÓN }\end{array}$} \\
\hline Escala & Claridad & Pertinencia & Suficiencia \\
\hline 1 & $0 \%$ & $0 \%$ & $0 \%$ \\
\hline 2 & $2.5 \%$ & $0 \%$ & $2.5 \%$ \\
\hline 3 & $7.5 \%$ & $0 \%$ & $2.5 \%$ \\
\hline 4 & $90.0 \%$ & $100 \%$ & $95.0 \%$ \\
\hline
\end{tabular}

Fuente: elaboración propia.
El capítulo 9. Desechos, quedó constituido por los aspectos de: tipos de desechos, flujo, contenedores, y disposición; conformado por un total de 6 requisitos y de acuerdo con la integración se obtuvo el siguiente resultado:

Figura 11. Resultados obtenidos en el capítulo 9. Desechos

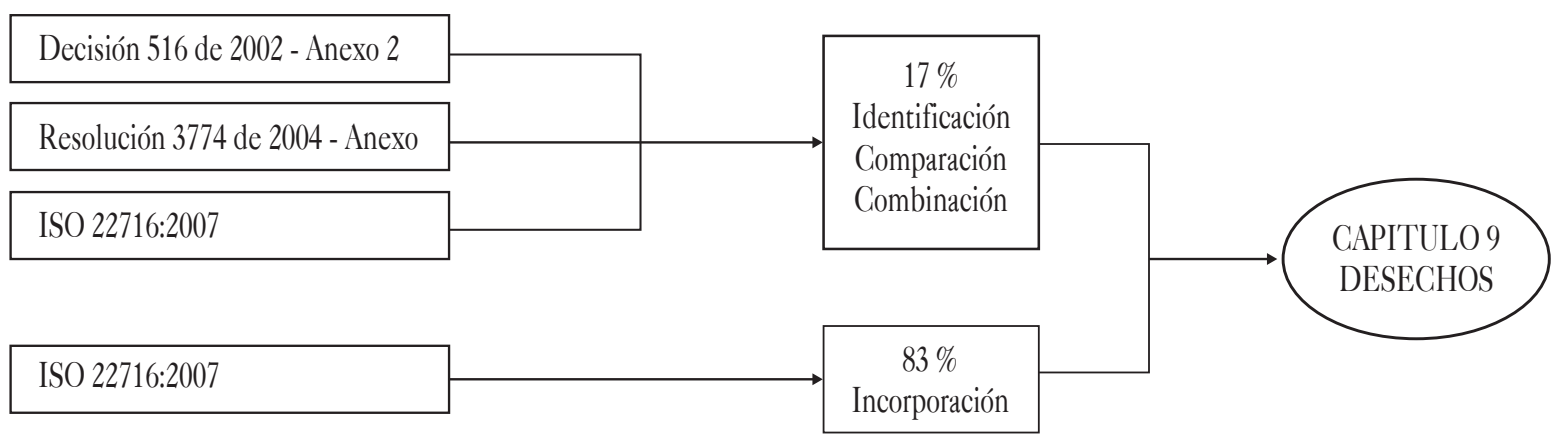

Fuente: elaboración propia.

Los resultados obtenidos de la evaluación por los expertos para este capítulo, se concentraron en la escala 4 para los criterios de claridad, pertinencia y suficiencia.

Tabla 10. Resultados obtenidos para el capítulo 9. Desechos

\begin{tabular}{|c|c|c|c|}
\hline \multicolumn{4}{|c|}{ CAPÍTULO 9. DESECHOS } \\
\hline Escala & Claridad & Pertinencia & Suficiencia \\
\hline 1 & $0 \%$ & $0 \%$ & $0 \%$ \\
\hline 2 & $6.3 \%$ & $4.2 \%$ & $0 \%$ \\
\hline 3 & $2.1 \%$ & $2.1 \%$ & $6.3 \%$ \\
\hline 4 & $91.7 \%$ & $93.8 \%$ & $93.8 \%$ \\
\hline
\end{tabular}

Fuente: elaboración propia.
El capítulo 10. Subcontratación, quedó constituido por los aspectos de: tipos de subcontratación, contratante, contratista, y contrato; conformado por un total de 11 requisitos y de acuerdo con la integración se obtuvo el siguiente resultado: 
Figura 12. Resultados obtenidos en el capítulo 10. Subcontratación

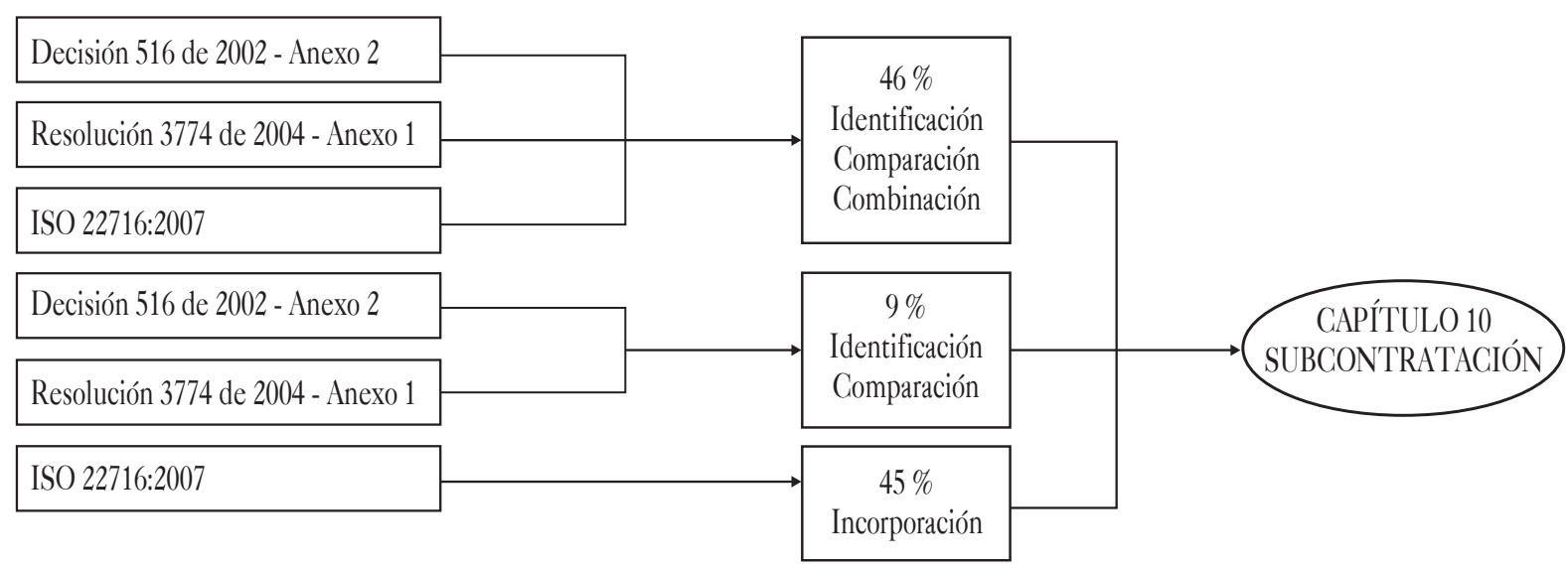

Fuente: elaboración propia.

Los resultados obtenidos de la evaluación por los expertos para este capítulo, se concentraron en la escala 4 para los criterios de claridad, pertinencia y suficiencia.

Tabla 11. Resultados obtenidos para el capítulo 10. Subcontratación

\begin{tabular}{|c|c|c|c|}
\hline \multicolumn{4}{|c|}{ CAPÍTULO 10. SUBCONTRATACIÓN } \\
\hline Escala & Claridad & Pertinencia & Suficiencia \\
\hline 1 & $0 \%$ & $12.5 \%$ & $12.5 \%$ \\
\hline 2 & $0 \%$ & $0 \%$ & $6.3 \%$ \\
\hline 3 & $12.5 \%$ & $0 \%$ & $0 \%$ \\
\hline 4 & $87.5 \%$ & $87.5 \%$ & $81.3 \%$ \\
\hline
\end{tabular}

Fuente: elaboración propia.

El capítulo 11. Desviaciones, quedó constituido por 2 requisitos, los cuales corresponden a los requisitos de la ISO 22716:2007, ya que la Decisión 516 de 2002 y la Resolución 3774 de 2004 no contemplan las desviaciones respecto a las especificaciones de calidad definidas.

Los resultados obtenidos por la evaluación de los expertos para este capítulo, se concentraron en la escala 4 para los criterios de claridad, pertinencia y suficiencia.
Tabla 12. Resultados obtenidos para el capítulo 11. Desviaciones

\begin{tabular}{|c|c|c|c|}
\hline \multicolumn{4}{|c|}{ CAPÍTULO 11. DESVIACIONES } \\
\hline Escala & Claridad & Pertinencia & Suficiencia \\
\hline 1 & $0 \%$ & $12.5 \%$ & $12.5 \%$ \\
\hline 2 & $0 \%$ & $0 \%$ & $6.3 \%$ \\
\hline 3 & $12.5 \%$ & $0 \%$ & $0 \%$ \\
\hline 4 & $87.5 \%$ & $87.5 \%$ & $81.3 \%$ \\
\hline
\end{tabular}

Fuente: elaboración propia.

El capítulo 12. Quejas y retiros, quedó constituido por los aspectos de: quejas sobre el producto y retiro del producto; conformado por un total de 11 requisitos, aunque la propuesta inicial que fue sometida a la evaluación por los expertos era de 12 requisitos, los cuales corresponden a los requisitos incorporados de la ISO 22716:2007, por lo que la Decisión 516 de 2002 y la Resolución 3774 de 2004 no contemplan las quejas sobre el producto ni retiro del producto.

Los resultados obtenidos por la evaluación de los expertos para este capítulo, se concentraron en la escala 4 para los criterios de claridad, pertinencia y suficiencia. 
Tabla 13. Resultados obtenidos para el capítulo 12. Quejas y retiros

\begin{tabular}{|c|c|c|c|}
\hline \multicolumn{4}{|c|}{ CAPÍTULO 12. QUEJAS Y RETIROS } \\
\hline Escala & Claridad & Pertinencia & Suficiencia \\
\hline 1 & $0 \%$ & $3.1 \%$ & $2.1 \%$ \\
\hline 2 & $10.4 \%$ & $2.1 \%$ & $6.3 \%$ \\
\hline 3 & $0 \%$ & $0 \%$ & $1.0 \%$ \\
\hline 4 & $89.6 \%$ & $94.8 \%$ & $90.6 \%$ \\
\hline
\end{tabular}

Fuente: elaboración propia.

El capítulo 13. Control de cambios, quedó constituido por 1 requisito el cual corresponde a la identificación, comparación y combinación del requisito presente en el numeral 8 del capítulo VIII del anexo 2 de la Decisión 516 de 2002, numeral 9 del capítulo VIII el anexo 1 de la Resolución 3774 de 2004 y numeral 15 de la ISO 22716:2007.

Los resultados obtenidos de la evaluación por los expertos se concentraron en la escala 4 para los criterios de claridad, pertinencia y suficiencia.
Tabla 14. Resultados obtenidos para el capítulo 13. Control de cambios

\begin{tabular}{|c|c|c|c|}
\hline \multicolumn{4}{|c|}{ CAPÍTULO 13. CONTROL DE CAMBIOS } \\
\hline Escala & Claridad & Pertinencia & Suficiencia \\
\hline 1 & $0 \%$ & $0 \%$ & $0 \%$ \\
\hline 2 & $12.5 \%$ & $12.5 \%$ & $12.5 \%$ \\
\hline 3 & $0 \%$ & $0 \%$ & $0 \%$ \\
\hline 4 & $87.5 \%$ & $87.5 \%$ & $87.5 \%$ \\
\hline
\end{tabular}

Fuente: elaboración propia.

El capítulo 14. Auditoría interna, quedó constituido por los aspectos de: enfoque y seguimiento; conformado por un total de 4 requisitos, aunque la propuesta inicial que fue sometida a la evaluación por los expertos era de 5 requisitos, y de acuerdo con la integración se obtuvo el siguiente resultado:

Fiǵura 13. Resultados obtenidos en el capítulo 14. Auditoría interna

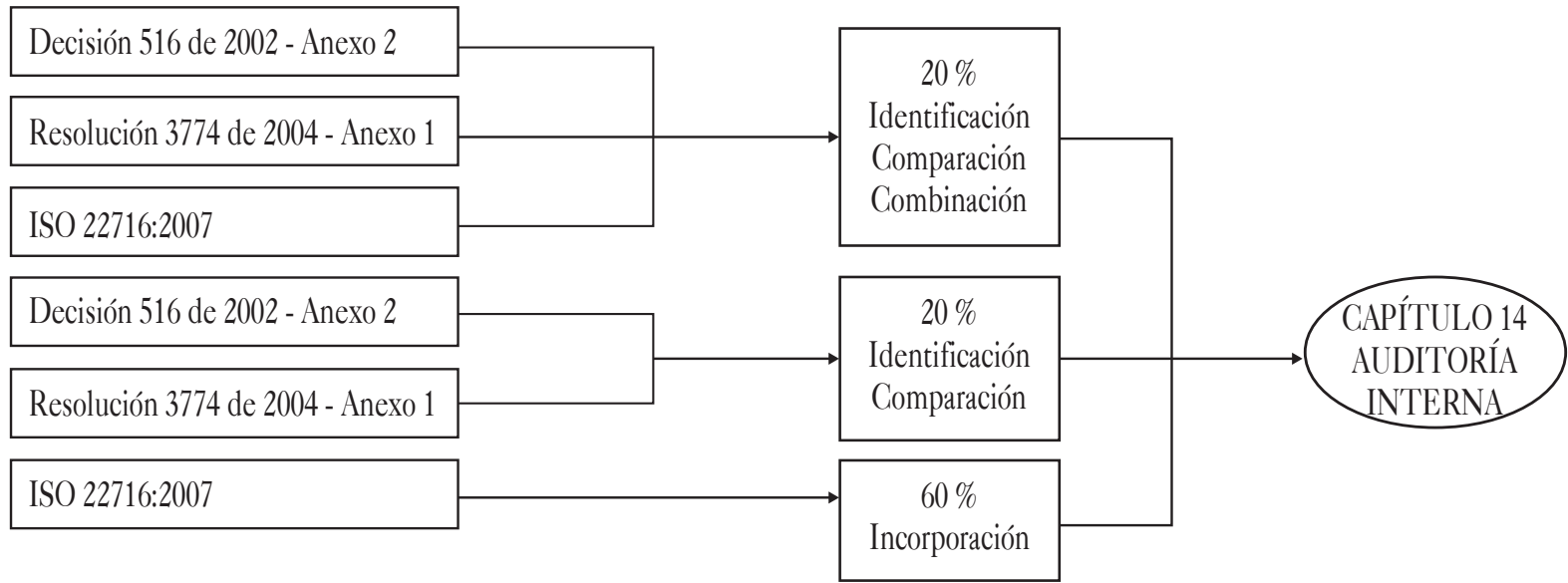

Fuente: elaboración propia. 
Los resultados obtenidos de la evaluación por los expertos para este capítulo, se concentraron en la escala 4 para los criterios de claridad, pertinencia y suficiencia.

Tabla 15. Resultados obtenidos para el capítulo 14. Auditoría interna

\begin{tabular}{|c|c|c|c|}
\hline \multicolumn{4}{|c|}{ CAPÍTULO 14. AUDITORÍA INTERNA } \\
\hline Escala & Claridad & Pertinencia & Suficiencia \\
\hline 1 & $0 \%$ & $2.5 \%$ & $2.5 \%$ \\
\hline 2 & $5.0 \%$ & $2.5 \%$ & $5.0 \%$ \\
\hline 3 & $0 \%$ & $0 \%$ & $5.0 \%$ \\
\hline 4 & $95.0 \%$ & $95.0 \%$ & $87.5 \%$ \\
\hline
\end{tabular}

Fuente: elaboración propia.
El capítulo 15. Documentación, quedó constituido por los aspectos de: tipos de documentos, redacción, aprobación y distribución, revisión y archivo; conformado por un total de 18 requisitos y de acuerdo con la integración se obtuvo el siguiente resultado:

Fig̉ura 14. Resultados obtenidos en el capítulo 15. Documentación

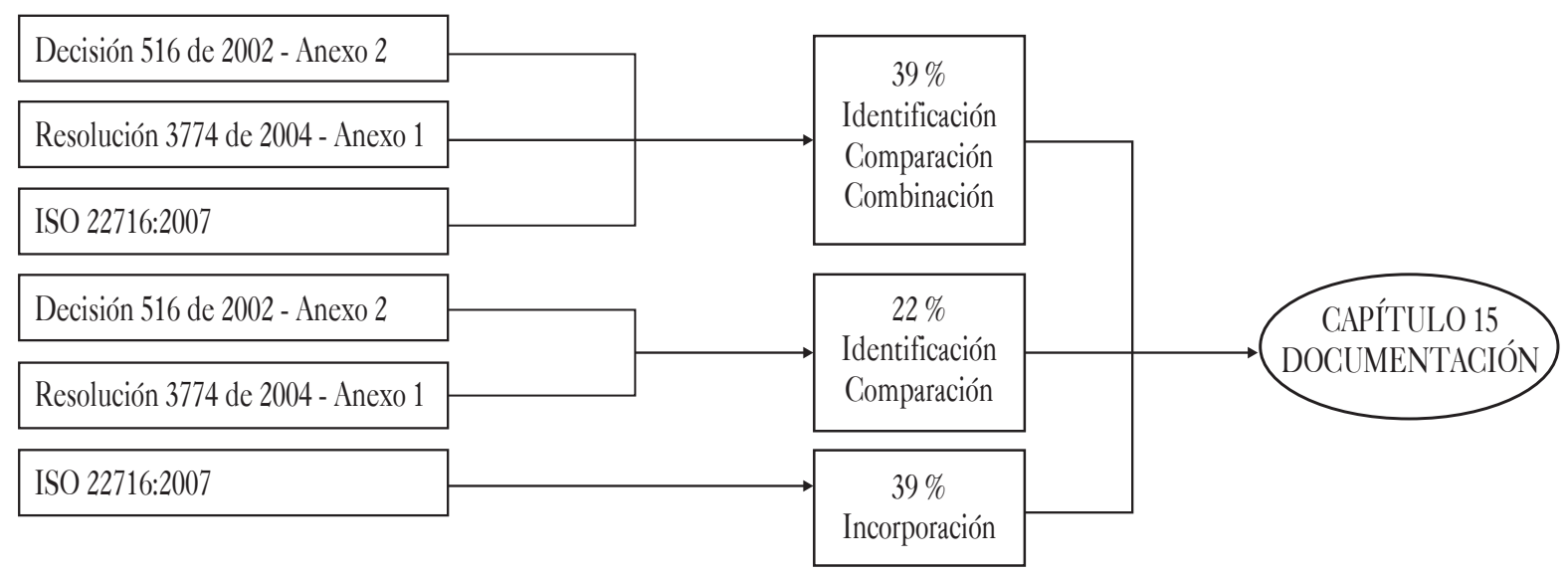

Fuente: elaboración propia.

Los resultados obtenidos de la evaluación por los expertos para este capítulo, se concentraron en la escala 4 para los criterios de claridad, pertinencia y suficiencia, tal como se muestra en la tabla 16 :
Tabla 16. Resultados obtenidos para el capítulo 15. Documentación

\begin{tabular}{|c|c|c|c|}
\hline \multicolumn{4}{|c|}{ CAPÍTULO 15. DOCUMENTACIÓN } \\
\hline Escala & Claridad & Pertinencia & Suficiencia \\
\hline 1 & $0 \%$ & $0.7 \%$ & $0.7 \%$ \\
\hline 2 & $4.2 \%$ & $2.8 \%$ & $3.5 \%$ \\
\hline 3 & $4.2 \%$ & $3.5 \%$ & $4.9 \%$ \\
\hline 4 & $91.7 \%$ & $93.1 \%$ & $91.0 \%$ \\
\hline
\end{tabular}

Fuente: elaboración propia. 
De acuerdo con el resultado del diseño de la propuesta, se procedió a realizar la integración de la Resolución 3773 de 2004, el anexo 2 de la Resolución 2004 y la propuesta integral para el sistema de gestión de las Buenas Prácticas de Manufactura del sector cosmético, creándose la Guía de Verificación de la Propuesta Integral del Sistema de Gestión para las Buenas Prácticas de Manufactura del Sector Cosmético en Colombia, la cual quedó constituida por un total de 218 requisitos.

\section{CONCLUSIONES}

De acuerdo con el fenómeno global que está ocurriendo, en cuanto a que la integración de los sistemas de gestión estandarizados traen beneficios como la mejora y el rendimiento, la reducción de la duplicación de tareas, la documentación, la eliminación de funciones y estructuras superpuestas, así como la reducción del tiempo y costos de auditorías (Ribeiro, Santos, Ferreira y Silva, 2017); se puede precisar que el diseño de la propuesta integral entre el anexo 2 de la Decisión 516 del 2002, la Resolución 3773 del 2004, la Resolución 3774 del 2004 y la norma ISO 22716:2007, trae para los fabricantes de productos cosméticos en Colombia la optimización de sus recursos, al momento de gestionar las Buenas Prácticas de Manufactura, cumpliendo simultáneamente con los requisitos de ley a nivel nacional como internacional.

El diseño de la propuesta integral quedó constituido por un total de 239 requisitos, de los cuales el $50 \%$ representan los requisitos incorporados de la norma ISO 22716:2007, en aspectos que no son contemplados por la legislación nacional en materia de la responsabilidad de la dirección, higiene personal como lavado de manos y prohibiciones de prácticas antihigiénicas, visitantes y personal no formado, pisos, paredes, techos, ventanas, pisos, insumos empleados en las actividades de mantenimiento de equipos e instalaciones, análisis de las materias primas, almacenamiento de producto a granel, devolución de las materias primas, los materiales de envase y empaque al almacén, despacho de productos terminados, tratamiento del producto que está fuera de especificación, desechos, quejas y retiros.

Mientras que el $17 \%$ representa los requisitos identificados y comparados de la Decisión 516 de 2002 y el anexo 1 de la Resolución 3774 de 2004, en los aspectos del perfil del director técnico, los exámenes médicos de ingreso y periódicos, la clasificación de las áreas (negras y grises), la exclusividad de las áreas para la fabricación de productos cosméticos, ubicación y dotación de los vestieres y los diferentes tipos de aires utilizados en la producción.

El $33 \%$ de los requisitos de la propuesta integral representan los requisitos que se identificaron, compararon y combinaron de la Decisión 516 de 2002 en su anexo 2, la Resolución 3774 de 2004 en su anexo 1 y la ISO 22716:2007.

En el análisis estadístico de las calificaciones obtenidas por los expertos, se empleó la correlación intraclase (ICC) en donde los resultados arrojaron poca significancia, por cuanto los resultados obtenidos fueron muy homogéneos y no se encontró varianza entre las calificaciones y los requisitos evaluados, por lo que no se empleó otro tipo de procedimiento estadístico.

\section{REFERENCIAS}

Agencia Valencia de Salut. (11 de julio de 2013). Edición comentada - Buenas Prácticas de fabricación de productos cosméticos. Recuperado de http://www. san.gva.es/documents/152919/5745148/10_Edici $\% \mathrm{C} 3 \% \mathrm{~B} 3 \mathrm{n}+\mathrm{comentada}+\mathrm{BPPC}+($ Edici\%C3\%B3n +Julio+2013).pdf 
Asociación Española de Normalización y Certificación (2005). Guía para la integración de los sistemas de gestión. UNE 66177. Madrid: AENOR.

Bernardo, M., Casadesus, M., Karapetrovic, S., \& Heras, I. (2009). How integrated are environmental, quality and other standardized management systems? An empirical study. Journal of Cleaner Production, 17(8), 742-750. Retrieved from https://www.sciencedirect.com/science/article/pii/ S0959652608002813

Bonilla, A., y Martínez, J. A. (2015). Propuesta de instrumento para medir el nivel de integración de los sistemas de gestión en organizaciones colombianas certificadas en ISO 9001, ISO 14001 y OHSAS 18001. (Tesis de maestría). Bogotá D. C.: Convenio Universidad Santo Tomás-ICONTEC.

Cámara de la Industria Cosmética y de Aseo de la ANDI. (2013). Desempeño económico y competitividad. Informe de sostenibilidad 2013, 34. Recuperado de https://issuu.com/agencia_central/docs/ informe_andi

Comisión de la Comunidad Andina. (15 de marzo de 2002). Armonización de legislaciones en materia de productos cosméticos (Decisión 516 de 2002). Recuperado de https://www.invima.gov.co/decisionescosmetico/detail.html

Conseil de l'Europe. (1995). Lignes directrices de bonnes pratiques de production des produits cosmétiques (BPPC). Germany : Conseil de l’Europe Publishing.

Consejo Nacional de Política Económica y Social. (23 de junio de 2008). II Marco Conceptual. Conpes 3527.
Hernández, R., Fernández, C., y Baptista, M. (2010). Metodología de la investigación 5. ed. México, D. F.: McGraw-Hill.

International Organization for Standardization. (2007). Cosmetics - Good Manufacturing Practices (GMP) Guidelines on Good Manufacturing Practices. (ISO 22716:2007). Retrieved from https://www.iso.org/ standard/36437.html

INVIMA. (2018a). Establecimientos certificados con capacidad de producción cosmética. Recuperado de https://www.invima.gov.co/a\%C3\%B102018-establecimientos-certificados-con-capacidad-de-producci\%C3\%B3n-cosm\%C3\%A9tica

INVIMA. (2018b). Establecimientos certificados con Buenas Prácticas de Manufactura. Recuperado de https://www.invima.gov.co/normatividadsp-510373846/alimentos/resoluciones-alimentos/ resoluciones-2002/322-direccion-de-cosmeticosaseo-plaguicidas-y-produ/establecimientos-autorizados-de-cosmeticos/3024-establecimientos-certificados-con-buenas-practicas-de-manufacturacosmetica.html

Llanes, M., Isaac, C. L., Moreno, M., y García, G. (2014). De la gestión por procesos a la gestión integrada por procesos. Revista Cubana Ingeniería Industrial, 35(3), 262.

Ministerio de Protección Social. (10 de noviembre de 2004a). Norma Técnica Armonizada de Buenas Prácticas de Manufactura Cosmética y la Guía de Verificación de Buenas Prácticas de Manufactura Cosmética. (Resolución 3774 de 2004). Recuperado de https://www.invima.gov.co/resoluciones-encosmeticos/detail.html 
Ministerio de Protección Social. (10 de noviembre de 2004b). Guía de capacidad para la fabricación de productos cosméticos. (Resolución 3773 de 2004). Recuperado de https://www.invima.gov.co/resoluciones-en-cosmeticos/detail.html

Programa Safe+, ONUD y Procolombia. (2016). Recomendaciones para exportar cosméticos a la Unión Europea. Recuperado de https://www.ptp.com.co/documentos/RECOMENDACIONES_SAFE.pdf
Ribeiro, F., Santos, G., Ferreira, M., \& Silva, R (2017). Integrated Management Systems: Trends for Portugal in the 2025 horizon. Procedia Mamufacturing, 13, 1192. Retrieved from https://ac.els-cdn.com/ S2351978917308338/1-s2.0-S2351978917308338main.pdf?_tid=9116b654-10b2-11e8-b78b00000aacb362\&acdnat=1518522164_d5bfdc6c555a0d96bbd674aa30734fcc 